\title{
THE KRAFT SUM AS A MONOTONE FUNCTION ON THE REFINEMENT-ORDERED SET OF UNIQUELY DECIPHERABLE CODES
}

\author{
STEPHAN FOLDES
}

\begin{abstract}
The set of all uniquely decipherable (UD) codes is partially ordered by refinement, meaning that all strings in the cruder code can be represented as concatenations of strings taken from the finer code. The Kraft sum is a monotone (increasing) function on this poset. In the refinement order, chains of UD codes having the same Kraft sum are necessarily of the simple descending type.
\end{abstract}

\section{INTRODUCTION}

Let $A$ be any non-empty finite set, called alphabet. Let con : $A^{* *} \longrightarrow A^{*}$ be the concatenation map which to every string of strings associates their concatenation. In this note we shall call any finite subset $C \subseteq A^{*}$ not containing the null string a code. A code is said to be uniquely decipherable (UD) if con is injective on the subset $C^{*}$ of $A^{* *}$. (Note that several authors, including Berstel and Perrin [1], reserve the term "code" to mean UD code.)

For any codes $C$ and $D$ write $C \leq D$ and say that $D$ is finer than $C$, or that it is a refinement of $C$, if $C \subseteq \operatorname{con}[D]$. This is a partial order relation (antisymmetry is ensured by unique decipherability). We say that $D$ is an irredundant refinement of $C$ if $C \leq D$ and no proper subset of $D$ is finer than $C$. Every code has infinitely many refinements. However, due to its finiteness, each code can have only finitely many irredundant refinements.

Denoting by $r$ the number of elements of the alphabet $A$, the Kraft sum $K(C)$ of any code $C \subseteq A^{*}$ is defined as $\sum_{\mathbf{x} \in C} r^{-\operatorname{len}(\mathbf{x})}$, where len is the length function. In [5] McMillan showed that if $C$ is a uniquely decipherable code, then its Kraft sum is at most 1 . Simplified combinatorial proofs were given by Karush [3], and by Berstel and Perrin ([1] Chapter 1, Theorem 4.2). The proof was also reformulated in [2] as an argument involving evaluations of polynomials with non-commuting indeterminates corresponding to the various (infinitely many) strings in $A^{*}$. In [2] we also concluded that for any UD codes $C$ and $D$ such that $C \leq D$, the inequality $K(C) \leq K(D)$ holds. Here the purely combinatorial proof due to Berstel and Perrin [1] is shown to yield the same conclusion that was reached in [2], and from

$M S C$ (2010): primary 05A20, 68P30, 38R05.

Keywords: code, unique decipherability, Kraft sum, Kraft inequality, MacMillan theorem.

This work has been co-funded by Marie Curie Actions and supported by the National Development Agency (NDA) of Hungary and the Hungarian Scientific Research Fund (OTKA, contract number 84593), within a project hosted by the University of Miskolc, Department of Analysis. 
this some further conclusions are drawn about the set of UD codes having the same Kraft sum.

The Kraft inequality as originally established by Kraft [4] stated that $K(C) \leq 1$ for instantaneous (prefix-free) codes, which are a special class of UD codes. This can be verified in several ways - for a recent approach, which also generalizes the inequality to data structures other than strings, see Valmari [6].

\section{Statements AND PROOFS}

For any code $C$ and positive integer $k$, denote by $C^{k}$ the code consisting of all possible concatenations of $k$ (not necessarily distinct) members of $C$. Note that if $C$ is a UD code, then $C^{k}$ is also UD and $\operatorname{Card}\left(C^{k}\right)=[\operatorname{Card}(C)]^{k}$. The following appears in Berstel and Perrin [1], Chapter 1, Proposition 4.1.

Proposition 2.1 ([1]). For any code $C$ over a given alphabet and positive integer $k$, we have $K\left(C^{k}\right) \leq K(C)^{k}$ and the following conditions are equivalent:

(i) $C$ is uniquely decipherable,

(ii) $K\left(C^{k}\right)=K(C)^{k}$ for all positive integers $k$.

Proof. (As given by Berstel and Perrin [1]). For each positive $k$, denote by $C^{(k)}$ the set of strings $\left(\mathbf{v}_{1}, \ldots, \mathbf{v}_{k}\right)$ of $k$ (not necessarily distinct) words from $C$. Clearly, $C^{(k)} \subseteq A^{* *}$.

We claim that $C$ is UD if and only if concatenation restricted to $C^{(k)}$ is an injective map for every $k$. These injectivity conditions are clearly necessary for $C$ to be UD. On the other hand, if $C$ is not UD, then, for some positive $m, n$, two different strings of words, $x=\left(\mathbf{x}_{1}, \ldots, \mathbf{x}_{m}\right) \in C^{(m)}$ and $y=\left(\mathbf{y}_{1}, \ldots, \mathbf{y}_{n}\right) \in C^{(n)}$ yield the same concatenation, $\operatorname{con} x=$ con $y$. Let $k=m+n$. The strings of words $\left(\mathbf{x}_{1}, \ldots, \mathbf{x}_{m}, \mathbf{y}_{1}, \ldots, \mathbf{y}_{n}\right)$ and $\left(\mathbf{y}_{1}, \ldots, \mathbf{y}_{n}, \mathbf{x}_{1}, \ldots, \mathbf{x}_{m}\right)$ are both in $C^{(k)}$, they are distinct, and they yield the same concatenation, proving the claim. $C^{k}$.

Observe that concatenation restricted to $C^{(k)}$ is always a surjective map onto

For $\left(\mathbf{v}_{1}, \ldots, \mathbf{v}_{k}\right) \in C^{(k)}$ the word $\operatorname{con}\left(\mathbf{v}_{1}, \ldots, \mathbf{v}_{k}\right)$ in $C^{k}$ contributes to the Kraft sum $K\left(C^{k}\right)$, a term equal to the product of the terms $r^{-\operatorname{len}\left(\mathbf{v}_{i}\right)}, 1 \leq i \leq k$ of the Kraft sum of $C$. Sum of these products over all members of $C^{(k)}$ equals $K(C)^{k}$ and it yields exactly $K\left(C^{k}\right)$ if the map con is injective on $C^{(k)}$, otherwise it yields a strict upper bound of $K\left(C^{k}\right)$.

We can apply to two codes comparable by refinement the reasoning presented in Berstel and Perrin's proof of Theorem 4.2 in Chapter 1 of [1]. Let $C$ be a UD code and let $D$ be any code finer than $C$. There is a positive integer $m$ such that for all integers $n>m, C$ is disjoint from $D^{n}$. For any fixed positive integer $k$, we have (by an obvious induction with respect to $k$ ) that

$$
C^{k} \subseteq D^{k} \cup D^{k+1} \cup \ldots \cup D^{m k}
$$


and $K(C)^{k}=K\left(C^{k}\right)$ is less than or equal to

$$
\begin{aligned}
& K\left(D^{k} \cup D^{k+1} \cup \ldots \cup D^{m k}\right) \\
\leq & K\left(D^{k}\right)+K\left(D^{k+1}\right)+\ldots+K\left(D^{m k}\right) \\
\leq & K(D)^{k}+K(D)^{k+1}+\ldots+K(D)^{m k} \\
= & K(D)^{k}\left[1+K(D)+\ldots+K(D)^{(m-1) k}\right] .
\end{aligned}
$$

First, when we choose the finest code $D$ consisting of all the words of length 1 , we get $K(C)^{k} \leq(m-1) k+1$. It follows that $K(C) \leq[(m-1) k+1]^{1 / k}$, for every $k$. Necessarily, $K(C) \leq 1$. Second, if we assume that code $D$ is also UD, and thus, its Kraft sum is at most 1, we get

$$
\left[\frac{K(C)}{K(D)}\right]^{k} \leq 1+K(D)+\ldots+K(D)^{(m-1) k} \leq(m-1) k+1
$$

which is true for all $k$, implying that the ratio on the left hand side is at most 1 , $K(C) \leq K(D)$. This yields the following extension of McMillan's Theorem:

Proposition 2.2. The Kraft sum is a monotone (increasing) function on the refinement-ordered set of uniquely decipherable codes. For each UD code $C$, there are only finitely many finer UD codes with the same Kraft sum.

The second statement follows from the fact, noted above, that a UD code $C$ has only finitely many irredundant refinements, and from the observation that for a code $D$ finer than $C$ that is not an irredundant refinement of $C$, the Kraft sums cannot be equal, the inequality between them has to be strict, $K(C)<K(D)$. By Proposition 2, every UD code $C$ has at least one UD refinement $D$ with the same Kraft sum that can no longer be properly refined without increasing the Kraft sum. As a further consequence, we have:

Proposition 2.3. In the refinement-ordered set of UD codes, all infinite chains of UD codes with the same Kraft sum are of type $\omega^{*}$ (i.e. of the same order type as the negative integers).

Infinite chains of UD codes all having the same Kraft sum exist indeed, in fact there is such a chain below every member of the poset of UD codes: for any UD code $C$, consider for example $C>C^{2}>C^{4}>\ldots>C^{2^{n}}>\ldots$.

Acknowledgement. The author wishes to thank S. Fegyverneki, S. Radeleczki, J. Szigeti and A. Valmari for useful comments and discussions.

\section{REFERENCES}

[1] J. Berstel and D. Perrin, Theory of Codes, Academic Press, 1985.

[2] S. Foldes, On McMillan's theorem about uniquely decipherable codes, ArXiv:0806.3277v2 (2008).

[3] J. Karush, A simple proof of an inequality of McMillan, IRE Trans. Information Theory IT-7 (1961), 118-118.

[4] L. G. Kraft, A Device for Quantizing, Grouping, and Coding Amplitude Modulated Pulses, Q.S. Thesis, MIT, 1949.

[5] B. McMillan, Two inequalities implied by unique decipherability, IRE Trans. Information Theory IT-2 (1956), 115-116. 
[6] A. Valmari, Does the Shannon bound really apply to data structures?, Proc. Estonian Acad. Sc. 62 (2013), 47-58.

Stephan Foldes, Department of Mathematics, Tampere University of Technology, 33101 Tampere, Finland

e-mail: sf@tut.fi
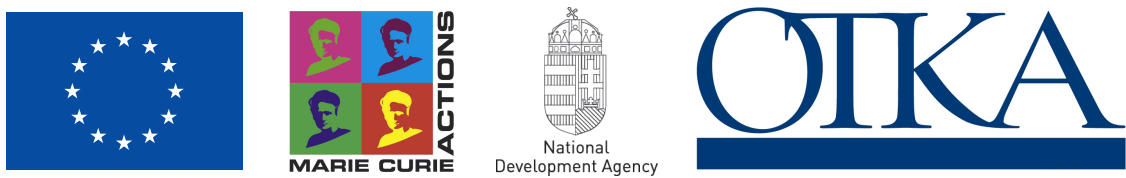